\title{
Effectiveness of Golimumab in Clinical Management of Patients with Rheumatoid Arthritis
}

\author{
Eisuke Shono
}

Published online: 4 April 2013

(C) The Author(s) 2013. This article is published with open access at Springerlink.com

\begin{abstract}
Background and Objectives Limited data are available regarding the use of golimumab $(100 \mathrm{mg})$ every 4 weeks, with or without methotrexate (MTX). The aim of this retrospective analysis was to evaluate the effectiveness and safety of golimumab following usual clinical practice in Japanese patients with rheumatoid arthritis (RA) according to the recommendations given in the Japanese package insert.

Patients and Methods Japanese RA patients with moderate-to-high disease activity, according to the 28-joint disease activity score based on C-reactive protein (DAS28CRP) criteria, despite treatment with MTX or another biological agent, were enrolled. Patients were assigned to $50 \mathrm{mg}$ golimumab plus MTX or $100 \mathrm{mg}$ golimumab monotherapy every 4 weeks for 24 weeks. All patients were given MTX if it was not contraindicated. The primary endpoint was the proportion of patients achieving clinical remission (defined as a DAS28-CRP $<2.3$ or a simplified disease activity index [SDAI] score $<3.3$ ) at 24 weeks.

Results Most patients received combined $50 \mathrm{mg}$ golimumab plus MTX (41/43). In these patients, the primary endpoint, clinical remission, was attained in $83 \%$ of patients according to DAS28-CRP criteria $(p<0.001)$ and $69 \%$ according to SDAI criteria $(p<0.001)$ by week 24 . Adverse events were reported in $11.6 \%$ of patients receiving golimumab.

Conclusions Golimumab (50 mg) plus MTX effectively reduced the signs and symptoms of RA and was generally well tolerated in patients with an inadequate response to MTX and other biological agents.
\end{abstract}

E. Shono $(\varangle)$

Shono Rheumatology Clinic, 1-10-27, Nishijin,

Sawara-ku, Fukuoka 814-0002, Japan

e-mail: shono-rheum@r2.dion.ne.jp

\section{Introduction}

In recent years, methotrexate (MTX) therapy at high dose levels and tumor necrosis factor (TNF) inhibitor therapy have been applied to treatment of rheumatoid arthritis (RA). Anti-TNF therapy, either alone or in combination with MTX (apart from infliximab, which should only be used in combination with MTX), is recommended in patients with active RA with inadequate response to MTX or another disease-modifying antirheumatic drug (DMARD) or combination of DMARDs or another antiTNF agent [1-3]. These new methods of treatment are expected to yield not only the alleviation of disease activity, but also structural improvement of the affected joints and improvement in daily life for patients. The three most widely used anti-TNF agents in Japan are infliximab, etanercept, and adalimumab, and numerous reports have been published on these agents [4-6].

Golimumab (GLM), a new human anti-TNF antibody agent created using transgenic mice, has been shown to exert effectiveness comparable to that of existing anti-TNF antibody agents when injected subcutaneously at 4-week intervals [7-13]. This drug was introduced in Japan in September 2011, thus providing a new treatment option for Japanese patients with RA. GLM can be administered either as monotherapy at a dosage of $100 \mathrm{mg}$ or in combination with MTX at dosages of 50 or $100 \mathrm{mg}$ every 4 weeks [14]. It is indicated not only in patients who have not previously received treatment with biological agents but also in patients who have experienced difficulties with infliximab or adalimumab therapy; for example, problems with neutralizing antibodies.

In Japan, there have been no published reports on the use of GLM in clinical practice to date. When patients are enrolled into clinical studies, age and disease activity are 
often taken into account to ensure safety and continued use of the investigational agent, so the populations studied differ from the population managed in real life. Therefore, this analysis evaluates the use of GLM in patients with RA receiving real-life clinical care at our clinic.

\section{Methods}

\subsection{Subjects}

This retrospective analysis included patients with baseline moderate-to-high disease activity according to a 28 -joint disease activity score based on C-reactive protein (DAS28CRP) $>3.2$ despite treatment with MTX or another biological agent. Patients had RA according to American College of Rheumatology (ACR) criteria [15] and had received GLM treatment as outpatients at our hospital, the Shono Rheumatology Clinic, Fukuoka, Japan, between September 2011 and April 2012. Consent in writing was obtained from each patient in advance.

\subsection{Treatment}

Patients received combination therapy with GLM plus MTX, with GLM administered at a dose of $50 \mathrm{mg}$ or $100 \mathrm{mg}$ every 4 weeks plus MTX administered at a dose of up to $8 \mathrm{mg} /$ week; or GLM monotherapy, with GLM administered at $100 \mathrm{mg}$ every 4 weeks, for a total of 24 weeks. All patients were prescribed MTX if it was not contraindicated. GLM was administered subcutaneously in accordance with the Japanese package insert [14].

\subsection{Outcome Measures}

The primary endpoint of this retrospective analysis of effectiveness was to evaluate the proportion of patients achieving remission defined as a DAS28-CRP $<2.3$ or a simplified disease activity index (SDAI) score $<3.3$. Mean changes in the DAS28-CRP from baseline to 4 weeks were also evaluated.

Safety was evaluated on the basis of adverse events and laboratory test data. For each parameter, additional stratified analyses were conducted, dividing the patients into two groups; that is, bio-naïve patients who had not received biological agents prior to receiving GLM, and patients who had received prior biological agents (i.e., those switching from other biological agents to GLM).

\subsection{Statistical Analysis}

All data were included for efficacy and safety analyses. The last observation carried forward (LOCF) method was used to allow for missing data. Comparison of groups was performed using the Student's $t$ test with statistical significance set at $p<0.05$.

\section{Results}

\subsection{Patient Baseline Demographics and Clinical Characteristics}

Of all patients studied, 18 were bio-naïve cases and 25 had received prior biological agents, including infliximab $(n=4)$, etanercept $(n=10)$, adalimumab $(n=6)$, and tocilizumab $(n=5)$. Of the 25 patients previously treated with biological agents, 19 had received one prior biological agent and 6 had received two or more agents.

Table 1 shows the baseline demographics and disease characteristics of the patients enrolled into the study. Patient characteristics were generally well balanced between bio-naïve patients and those who had received a prior biological agent, except the proportion of women was slightly greater ( 96.0 vs $83.3 \%$ ) and disease duration was slightly longer (122.6 vs 105.3 months) in the bio-switching group.

\subsection{Interventions}

In total, 41 patients received GLM at a dose of $50 \mathrm{mg}$ every 4 weeks in combination with MTX (mean dose $6.23 \mathrm{mg} /$ week) and 2 patients received GLM monotherapy at a dose of $100 \mathrm{mg}$ every 4 weeks. Four patients were unsatisfied with the inconvenience of self-injection and injection pain of prior biological treatment, despite sufficient clinical response; therefore, those patients in clinical remission at baseline were switched to GLM treatment as a result of patients' wishes. Of the 43 patients, 35 completed the 24-week treatment period.

\subsection{Effectiveness}

Remission rates, defined as the proportion of patients achieving a DAS28-CRP $<2.3$ and an SDAI score $<3.3$, steadily improved over the course of treatment with GLM (Fig. 1). After 8 weeks of treatment, $71.4 \%$ of patients achieved a DAS28-CRP $<2.3$ and $50.0 \%$ achieved an SDAI score $<3.3$. After 8 weeks of treatment, the DAS28CRP and SDAI remission rates were higher in patients who had not received prior biological agents versus those who had (55.6 vs $50.0 \%$ and 61.1 vs $41.7 \%$, respectively).

The mean DAS28-CRP 4 weeks after the start of treatment was significantly improved compared with the pretreatment score [mean DAS28-CRP at week $4=1.80$ vs 4.14 (range 1.28-7.04) at baseline; $p<0.001$ ]. 
Table 1 Baseline demographics and disease characteristics in bio-naïve patients and patients who had received prior biological agents

\begin{tabular}{llll}
\hline & Total $(n=43)$ & Bio-naïve $(n=18)$ & Prior biologicals $(n=25)$ \\
\hline Sex $[n(\%)]$ & & & $24(96.0)$ \\
$\quad$ Female & $39(90.7)$ & $15(83.3)$ & $1(4.0)$ \\
$\quad$ Male & $4(9.3)$ & $3(16.7)$ & $61.4(32-76)$ \\
Age [years] & $59.1(32-79)$ & $55.8(37-79)$ & $122.6(12-252)$ \\
Disease duration [months] & $115.3(7-708)$ & $105.3(7-708)$ & $4.12(1.28-7.04)$ \\
DAS28-CRP & $4.14(1.28-7.04)$ & $4.16(2.61-6.39)$ & $22.20(2.81-62.30)$ \\
SDAI & $22.2(2.81-62.30)$ & $22.30(6.70-56.29)$ & $20.90(2.50-60.90)$ \\
CDAI & $20.92(2.50-60.90)$ & $20.94(6.50-56.00)$ & $6.0(0-24)$ \\
Tender joint count $[0-68]$ & $6.3(0-24)$ & $6.7(1-22)$ & $4.8(0-22)$ \\
Swollen joint count $[0-68]$ & $5.9(0-22)$ & $5.4(0-18)$ & $0.72(0-2)$ \\
mHAQ [0-24] & $0.65(0-2)$ & $0.44(0-2)$ & $1.4(0.1-8.4)$ \\
CRP [mg/dL] & $1.5(0.1-13.5)$ & $1.6(0.1-13.5)$ & $21(84.0)$ \\
RF positive $[n(\%)]$ & $34(79.0)$ & $13(72.2)$ & $11(44.0)$ \\
ACPA positive $[n(\%)]$ & $22(51.1)$ & $11(61.1)$ & \\
\hline All valis & &
\end{tabular}

All values are presented as means with ranges given in parentheses unless specified otherwise

$A C P A$ anti-citrullinated protein autoantibody, $C D A I$ clinical disease activity index, $C R P$ C-reactive protein, DAS28-CRP disease activity score 28 based on C-reactive protein, $m H A Q$ modified health assessment questionnaire; $R F$ rheumatoid factor, $S D A I$ simplified disease activity index

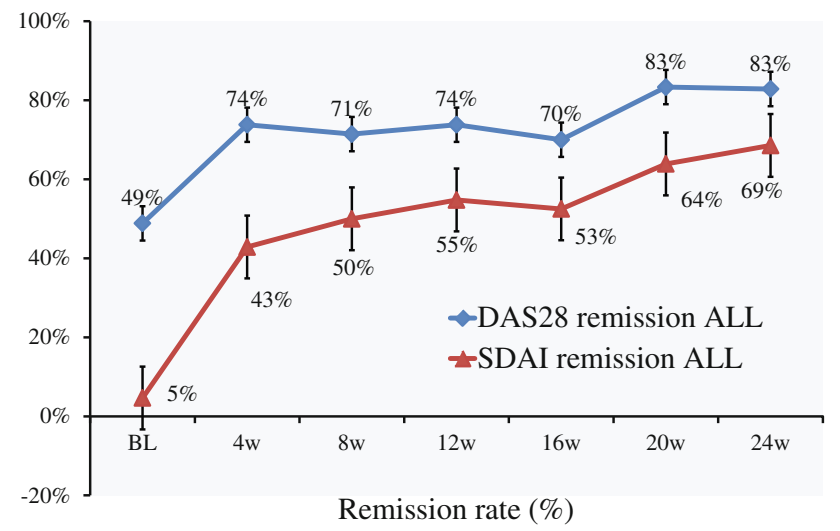

Fig. 1 Remission rate in 43 patients with rheumatoid arthritis treated with golimumab alone or in combination with methotrexate. Remission was defined as a 28-joint disease activity score based on C-reactive protein (DAS28-CRP) of $<2.3$ or a simplified disease activity index (SDAI) score of $<3.3$. DAS28-CRP remission and DAS28-CRP plus SDAI remission (ALL) are shown. $B L$ baseline, $W$ weeks

Improvements in DAS28-CRP and SDAI scores throughout the treatment period were similar in bio-naïve patients and those who had received prior biological agents (Figs. 2, 3). Changes in DAS28-CRP and SDAI scores at 4 and 8 weeks were statistically significant compared with baseline in both bio-naïve patients and those who had received prior biological agents (all $p<0.001$ ).

\subsection{Tolerability}

GLM was generally well tolerated with no unexpected safety issues observed. Adverse events (shown in Table 2) were reported in five patients, most of whom were receiving GLM $(50 \mathrm{mg}$ ) in combination with MTX (6 or $8 \mathrm{mg}$ ). Two patients reported fractures (one ankle and one femur); one patient was hospitalized due to renal impairment, chest pain, dyspnea, bronchial asthma, acute upper respiratory tract inflammation, and bronchitis; one patient (treated with GLM monotherapy at $100 \mathrm{mg}$ ) experienced venous thromboembolism and lower limb edema; and one patient reported renal impairment, hepatic function, and nephrogenic anemia. Consistent with other GLM safety data reported in Japanese clinical trials, no unknown adverse event was reported in this clinical analysis. All adverse events were resolved with treatment.

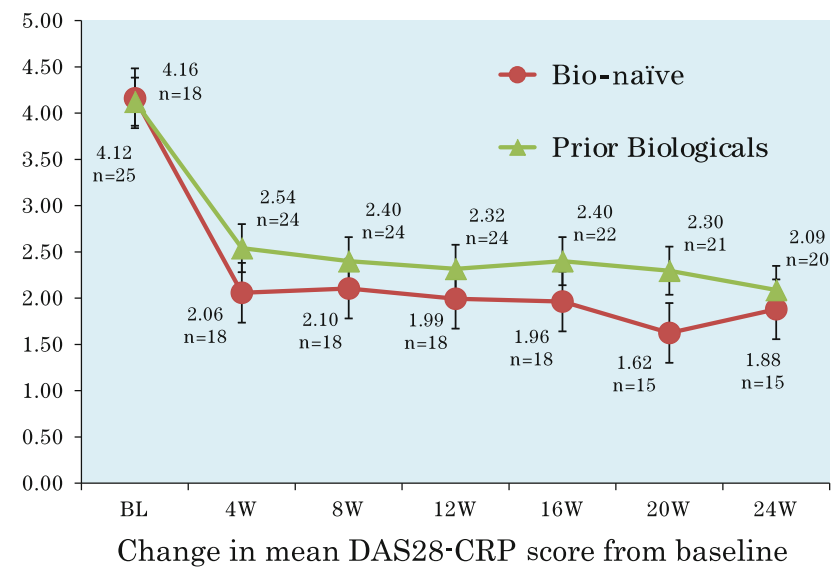

Fig. 2 Changes in mean 28-joint disease activity score based on $\mathrm{C}$-reactive protein (DAS28-CRP) in bio-naïve or previously treated patients with rheumatoid arthritis receiving golimumab alone or in combination with methotrexate. The dashed line represents the defined remission cutoff value of 2.3. BL baseline, $W$ weeks 


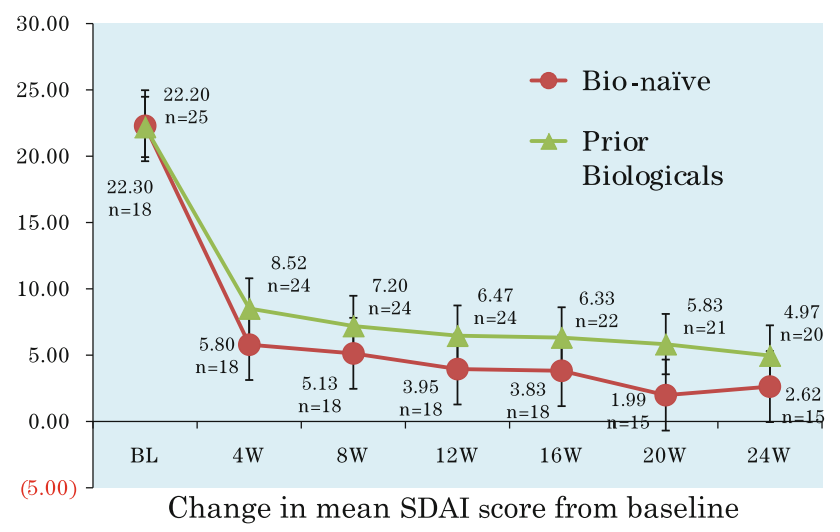

Fig. 3 Changes in mean simplified disease activity index (SDAI) score in bio-naïve or previously treated patients with rheumatoid arthritis receiving golimumab alone or in combination with methotrexate. The dashed line represents the defined remission cutoff value of 3.3. $B L$ baseline, $W$ weeks

\section{Discussion}

The present analysis in Japanese patients with RA in reallife clinical care revealed high effectiveness and safety of GLM alone or in combination with MTX, with significant improvements in mean DAS28-CRP and SDAI scores observed in bio-naïve patients 16 weeks after the start of treatment $(p<0.001)$. The reason for the high remission rate was considered to be the difference in average patient body weight between western countries and Japan (75 vs $50 \mathrm{~kg}$, respectively).

These effectiveness data are consistent with efficacy data from clinical studies [7-10, 12, 13, 16]. Most GLM studies are designed to permit rescue of patients at 16 weeks with alternative pharmacological therapy for those meeting the nonresponse criteria for early escape [8-10, 12, 13].

Similar to the GO-FORTH study [13], our clinical analysis involved patients treated with MTX at $8 \mathrm{mg} / \mathrm{week}$, which is the maximum dose approved in Japan at the time

Table 2 Adverse events and course reported in five patients with rheumatoid arthritis treated with golimumab every 4 weeks for 24 weeks

\begin{tabular}{lll}
\hline Case & Adverse events & Course \\
\hline 1 & Ankle fracture & $\begin{array}{c}\text { Treated by } \\
\text { another clinic }\end{array}$ \\
2 & Femur fracture & $\begin{array}{c}\text { Treated by } \\
\text { another clinic }\end{array}$ \\
3 & $\begin{array}{l}\text { Renal impairment, chest pain, dyspnea, } \\
\text { asthma bronchial, acute upper respiratory } \\
\text { tract inflammation, bronchitis }\end{array}$ & $\begin{array}{c}\text { Recovered as } \\
\text { inpatient }\end{array}$ \\
4 & $\begin{array}{c}\text { Embolism venous, edema lower limb } \\
\text { 5enal impairment, hepatic function }\end{array}$ & $\begin{array}{c}\text { Resolved, in } \\
\text { remission }\end{array}$ \\
& disorder, nephrogenic anemia & Recovered \\
\hline
\end{tabular}

that the patients were receiving treatment [17]. This is lower than the current recommended MTX dose in RA [3, $14,18]$ and lower than the MTX dose used in combination with GLM in other published studies [7, 9, 10]. Despite the low doses of MTX used, overall remission rates with GLM were high. Evidence suggests that, for patients receiving MTX who fail to achieve clinical remission based on SDAI or disease activity score scales, increasing the MTX dose needs to be considered at this time [17]. In the GO-FORWARD study, GLM was shown to be effective in patients who showed lower responses or who were refractory to prior MTX therapy $[9,10]$.

In the present retrospective analysis, manifestation of effectiveness appeared to be delayed in the bio-switching group compared with the bio-naïve group, suggesting the necessity for longer follow-up when evaluating effectiveness in patients who switch between biological therapies.

In a post-hoc analysis of the effectiveness in relation to the reasons for switching, the effectiveness did not differ significantly according to the reason (data not shown). This suggests that patients undergoing switching will respond to this therapy, regardless of the reasons for switching. This supports findings by Smolen et al. [12] that switching from other anti-TNF agents to GLM was effective regardless of the reasons for switching, indicating that GLM can serve as the second anti-TNF agent when patients are switched from another TNF agent. Of the five anti-TNF agents available, including certolizumab pegol, all have different affinities to TNF- $\alpha$; therefore, switching from one anti-TNF agent to another is likely to be effective.

Expression of antibodies to anti-TNF antibody agents such as infliximab, adalimumab, and certolizumab pegol monotherapy is not uncommon; however, incidences of anti-GLM antibodies in the GO-FORWARD [9] and GOFORTH [13] studies were remarkably low. Because GLM is prepared by the transgenic mouse technique, it is an antibody with high affinity for the antigen [19], which means that formation of unstable proteins or aggregations, which can serve as immunogens, is unlikely. Studies of GLM (100 mg) monotherapy were conducted in Caucasian and South American countries in GO-FORWARD [9, 10] and in Japan in GO-FORTH [13] and GO-MONO [16], and showed that GLM is an appropriate biological agent for preventing the loss of effectiveness in Caucasian, South American, and Japanese populations receiving long-term RA treatment $[9,10,13,16]$. As a result of findings from the GO-FORTH [13] and GO-MONO [16] studies, in addition to the 50-mg dose, GLM $(100 \mathrm{mg})$ every 4 weeks-as monotherapy and in combination with MTX - has been approved in Japan. Further studies at this dose level in larger numbers of patients are necessary.

Apart from the usual limitations relating to observational data and retrospective analyses, particularly with 
regard to selection and enrolment bias, a major limitation of our analysis is the small patient numbers, especially for patients receiving GLM $(100 \mathrm{mg})$ monotherapy. In addition, evaluation of levels of anti-GLM antibodies and the effects of GLM on structural joint damage in this real-life setting would have been useful; however, this was not evaluated in the original study.

Despite suppression of joint destruction being an important factor in improving the quality of life of patients with RA, the number of published reports evaluating this effect in patients treated with GLM is small, because of its relatively recent development. Emery et al. [7] reported that treatment with GLM suppressed joint destruction significantly 52 weeks after the start of treatment, and further long-term observation is needed. However, due to the short follow-up period in our analysis, such observation was not possible.

In the present analysis, there were no serious adverse events arising from the use of GLM, although deterioration in renal function was reported in two patients. An association with the development of malignant tumors has been suggested with GLM, and further clinical confirmation is warranted [20]. However, long-term observation of the patients in our study is needed before any definite conclusions can be made.

It is important to select a type of biological agent taking into account the lifestyle of individual patients. Despite reported problems with pain and administration site reactions, subcutaneous injection of drugs offers greater convenience than intravenous infusion, which requires physical immobilization for many hours at a hospital, and a longer dosing interval is also advantageous. Because GLM contains only small amounts of stimulating acidic additives and requires only a small volume of dosing solution, reported incidences of pain and administration site reactions are low [14].

\section{Conclusion}

In the present analysis, GLM plus MTX or GLM monotherapy used in clinical practice in Japanese patients with RA was confirmed to have high effectiveness and safety, comparable with existing biological agents. Thus, we conclude that GLM is a promising new alternative for the treatment of RA in Japanese patients showing poor response, those in whom the use of other biological agents is contraindicated, and cases where the use of MTX in combination with biological agents is difficult.

Acknowledgments Technical editing and manuscript styling was provided by Andrea Bothwell and post-submission editorial assistance was provided by Mary Hines, inScience Communications, Springer Healthcare, with funding provided by Janssen, Japan.
Conflict of Interest The author has no conflicts of interest to declare.

Open Access This article is distributed under the terms of the Creative Commons Attribution Noncommercial License which permits any noncommercial use, distribution, and reproduction in any medium, provided the original author(s) and the source are credited.

\section{References}

1. Agarwal SK. Biologic agents in rheumatoid arthritis: an update for managed care professionals. J Manag Care Pharm. 2011;17(9 Suppl B):S14-8.

2. Singh JA, Furst DE, Bharat A, et al. 2012 update of the 2008 American College of Rheumatology recommendations for the use of disease-modifying antirheumatic drugs and biologic agents in the treatment of rheumatoid arthritis. Arthritis Care Res (Hoboken). 2012;64(5):625-39.

3. Smolen JS, Landewe R, Breedveld FC, et al. EULAR recommendations for the management of rheumatoid arthritis with synthetic and biological disease-modifying antirheumatic drugs. Ann Rheum Dis. 2010;69(6):964-75.

4. Kievit W, Fransen J, Adang EMM, et al. Long-term effectiveness and safety of TNF-blocking agents in daily clinical practice: results from the Dutch Rheumatoid Arthritis Monitoring register. Rheumatology (Oxford). 2011;50(1):196-203.

5. Malottki K, Barton P, Tsourapas A, et al. Adalimumab, etanercept, infliximab, rituximab and abatacept for the treatment of rheumatoid arthritis after the failure of a tumour necrosis factor inhibitor: a systematic review and economic evaluation. Health Technol Assess. 2011;15(14):1-278.

6. Rahman MU, Buchanan J, Doyle MK, et al. Changes in patient characteristics in anti-tumour necrosis factor clinical trials for rheumatoid arthritis: results of an analysis of the literature over the past 16 years. Ann Rheum Dis. 2011;70(9):1631-40.

7. Emery P, Fleischmann R, van der Heijde D, et al. The effects of golimumab on radiographic progression in rheumatoid arthritis: results of randomized controlled studies of golimumab before methotrexate therapy and golimumab after methotrexate therapy. Arthritis Rheum. 2011;63(5):1200-10.

8. Emery P, Fleischmann RM, Moreland LW, et al. Golimumab, a human anti-tumor necrosis factor alpha monoclonal antibody, injected subcutaneously every four weeks in methotrexate-naive patients with active rheumatoid arthritis: twenty-four-week results of a phase III, multicenter, randomized, double-blind, placebo-controlled study of golimumab before methotrexate as first-line therapy for early-onset rheumatoid arthritis [Erratum appears in Arthritis Rheum. 2010;62(10):3005]. Arthritis Rheum. 2009;60(8):2272-83.

9. Keystone E, Genovese MC, Klareskog L, et al. Golimumab in patients with active rheumatoid arthritis despite methotrexate therapy: 52-week results of the GO-FORWARD study [Erratum appears in Ann Rheum Dis. 2011;70(1):238-9]. Ann Rheum Dis. 2010;69(6):1129-35.

10. Keystone EC, Genovese MC, Klareskog L, et al. Golimumab, a human antibody to tumour necrosis factor alpha given by monthly subcutaneous injections, in active rheumatoid arthritis despite methotrexate therapy: the GO-FORWARD Study [Erratum appears in Ann Rheum Dis. 2011;70(1):238]. Ann Rheum Dis. 2009;68(6):789-96.

11. Shealy D, Cai A, Staquet K, et al. Characterization of golimumab, a human monoclonal antibody specific for human tumor necrosis factor alpha. MAbs. 2010;2(4):428-39. 
12. Smolen JS, Kay J, Doyle MK, et al. Golimumab in patients with active rheumatoid arthritis after treatment with tumour necrosis factor alpha inhibitors (GO-AFTER study): a multicentre, randomised, double-blind, placebo-controlled, phase III trial [Erratum appears in Lancet. 2009;374(9699):1422]. Lancet. 2009; 374(9685):210-21.

13. Tanaka Y, Harigai M, Takeuchi T, et al. Golimumab in combination with methotrexate in Japanese patients with active rheumatoid arthritis: results of the GO-FORTH study. Ann Rheum Dis. 2012;71(6):817-24.

14. Janssen Pharmaceutical KK, Mitsubishi Tanabe Pharma Corporation. Simponi: Package insert. Japan 2011.

15. Aletaha D, Neogi T, Silman AJ, et al. 2010 Rheumatoid arthritis classification criteria: an American College of Rheumatology/ European League Against Rheumatism collaborative initiative. Arthritis Rheum. 2010;62(9):2569-81.

16. Takeuchi T, Harigai M, Tanaka Y, et al. Golimumab monotherapy in Japanese patients with active rheumatoid arthritis despite prior treatment with disease-modifying antirheumatic drugs: results of the phase $2 / 3$, multicentre, randomised, doubleblind, placebo-controlled GO-MONO study through 24 weeks. Ann Rheum Dis. Epub 2012 Sep 18.

17. Seto Y, Tanaka E, Inoue E, et al. Studies of the efficacy and safety of methotrexate at dosages over $8 \mathrm{mg} /$ week using the IORRA cohort database. Mod Rheumatol. 2011;21(6):579-93.

18. Electronic Medicines Compendium (eMC). Methotrexate $5 \mathrm{mg}$ tablets: Summary of prescribing information. 2012. http://www. medicines.org.uk/emc/medicine/22954/SPC\#POSOLOGY. Accessed 2013 Mar 21.

19. Hutas G. Golimumab as the first monthly subcutaneous fully human anti-TNF-alpha antibody in the treatment of inflammatory arthropathies. Immunotherapy. 2010;2(4):453-60.

20. Zidi I, Bouaziz A, Mnif W, et al. Golimumab and malignancies: true or false association? Med Oncol. 2011;28(2):641-8. 